\title{
miR-26a/b Inhibit Tumor Growth and Angiogenesis by Targeting the HGF-VEGF Axis in Gastric Carcinoma
}

\author{
Yiran Sia Haiyang Zhang ${ }^{a}$ Tao Ning ${ }^{a}$ Ming Bai ${ }^{a}$ Yi Wang ${ }^{a}$ Haiou Yang ${ }^{a}$

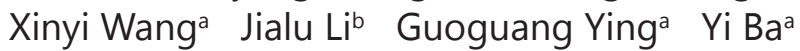 \\ aTianjin Medical University Cancer Institute and Hospital, National Clinical Research Center for Cancer, \\ Key Laboratory of Cancer Prevention and Therapy, Tianjin's Clinical Research Center for Cancer, \\ ${ }^{b}$ Department of Gastroenterology, Tianjin First Center Hospital, Tianjin, China
}

\section{Key Words}

MiR-26a/b • HGF • VEGF • Gastric carcinoma • Tumor growth • Angiogenesis

\begin{abstract}
Background/Aims: Abnormal expression of HGF is found in various cancers and correlates with tumor proliferation, metastasis and angiogenesis. However, the regulatory mechanism of the HGF-VEGF axis remains unclear. Methods: The expression characteristic of HGF in human gastric cancer tissues was shown by an immunohistochemistry assay, and the expression levels of target protein were detected by Western blot. The relative levels of $\mathrm{miR}-26 \mathrm{a} / \mathrm{b}$ and target mRNA were examined by qRT-PCR. We used bioinformatics tools to search for miRNAs that can potentially target HGF. A luciferase assay was used to confirm direct targeting. Furthermore, the functions of miR-26a/b and HGF were evaluated by cell proliferation and migration assays in vitro and by the mouse xenograft tumor model in vivo. Results: We found that the HGF protein was clearly increased while miR-26a/b were dramatically down-regulated in gastric cancer. miR-26a/b directly bind to the $3^{\prime}$-UTR of HGF mRNA at specific targeting sites. We demonstrated that the repression of the HGFVEGF pathway by miR-26a/b overexpression suppressed gastric cancer cell proliferation and migration. Furthermore, miR-26a/b also showed an anti-tumor effect in the xenograft mouse model by suppressing tumor growth and angiogenesis. Conclusions: miR-26a/b could suppress tumor tumorigenesis and angiogenesis by targeting the HGF-VEGF axis and could serve as a potential treatment modality for targeted therapy in the clinical treatment of gastric cancer.
\end{abstract}




\section{Introduction}

Gastric cancer (GC) ranks fifth for cancer incidence and second for cancer deaths. Approximately, $42.6 \%$ of all new cases originate in China [1]. GC is the leading cause of cancer-related mortality across the world, with a median overall survival of $\leq 12$ months for advanced stage GC [2]. The properties of high malignancy and early metastasis in GC should never be neglected; they are the significant reasons for the poor prognosis of GC. In addition, $70 \%$ of GC patients are first diagnosed at late stages with locally advanced or metastatic disease, which precludes the opportunity for surgical resection [3]. Despite progress in the treatment of GC, the effects of conventional therapies for GC, including chemotherapy and radiation, on prolonging survival are limited. Meanwhile, several molecular biomarkers, such as CEA, CA72-4 and CA19-9, have been used in clinical practice; however, none of these markers is highly specific for GC [4]. In recent years, along with studies of the tumor cell signal pathways, targeted medicine has become a promising strategy for the treatment of GC. Hence, studies on the novel pathways of GC tumorigenesis provide potential candidates to improve the prognosis of GC.

Hepatocyte growth factor (HGF) is a well-known peptide that performs various biological activities via the c-Met receptor. C-Met is a key member of the receptor tyrosine kinases (RTK) super-family and is expressed on normal hepatocytes, gastric epithelium; etc. [5]. The HGF/c-Met pathway contributes to normal embryo formation and tissue regeneration in physiological conditions. In pathological circumstances, HGF can promote the proliferation, migration, and metastasis of tumor cells via paracrine and autocrine effects [6]. The tyrosine phosphorylation of c-Met can elicit the activation of AKT, MAPK, and RAP1 in cancer cells, which are essential for cell cycle progression $[7,8]$. For the inhibition of $\mathrm{HGF} / \mathrm{c}-\mathrm{Met}$ activation in cancer patients, various c-Met antagonist and c-Met kinase inhibitors are now in clinical trials [8]. High expression of HGF/c-Met has been confirmed in GC tissues. Wu CW et al. concluded that a high positive rate of HGF (87.0\%) and HGF/cMet receptors (82.6\%) was present in intestinal-type GC [9], indicating that the inhibition of HGF/c-Met plays a significant role in treating GC $[2,10,11]$. HGF also plays a prominent role in inducing vascular endothelial growth factor (VEGF) expression [12,13]. VEGF is a key factor involved in tumor vascularization. As is showed in Fig. 2F, several studies have found that HGF/c-Met signaling turns on the angiogenic switch by targeting MAPK, PIK3 and Stat3 to up-regulate VEGF expression in tumor cells $[14,15]$. Thus, HGF/c-Met signaling operates as a key switch by turning on VEGF to invoke angiogenesis in cancer cells. Although the roles of HGF and VEGF have been discovered, the mechanisms underlying the up-regulation of HGF expression, especially in the tumorigenesis of GC, remain largely unknown.

microRNAs (miRNAs) are a class of small noncoding RNAs ( $\sim 22 \mathrm{bp}$ ) that are believed to regulate gene expression by directly binding with the 3'UTR of target mRNAs, causing translational inhibition or mRNA degradation [16]. Some miRNAs are closely related to the tumor formation [17-19]. They play the role of oncogenes or tumor suppressor genes by regulating the transcription and translation of downstream target genes. The miR-26 family, including miR-26a-1, miR-26a-2 and miR-26b, is widely expressed in many tissues. These 3 subtypes are located in chromosomes 3,12 and 2, respectively [20]. Recent studies have found that miR-26a/b are tumor suppressor genes in GC [21, 22]. However, latent mechanisms require further exploration to clarify the functions of miR-26a/b in GC tumorigenesis.

In this study, we determined that miR-26a/b were down-regulated; while HGF expression was significantly enhanced in GC tissues. The luciferase assay showed that HGF was a direct target of miR-26a/b. Overexpression of miR-26a/b in GC cells led to the inhibition of HGF and VEGF, thus inhibiting cell proliferation, migration and angiogenesis. The in vivo experiments demonstrated that the high level of miR-26a/b clearly inhibited tumor growth, while overexpression of HGF strongly accelerated tumor growth. Our results suggested that miR-26a and miR-26b were tumor suppressors by targeting HGF in GC. Therefore, a novel pathway comprising miR-26a/b, HGF and VEGF in GC may become a potential target for future clinical use. 


\section{Cellular Physiology Cell Physiol Biochem 2017;42:1670-1683 \\ \begin{tabular}{ll|l} 
and Biochemistry Published onlIne: July 24, 2017 & $\begin{array}{l}\text { (C) } 2017 \text { The Author(s). Published by S. Karger AG, Basel } \\
\text { www.karger.com/cpb }\end{array}$
\end{tabular}}

Si et al.: Novel Cancer Suppressive miRNAs in Gastric Carcinoma

\section{Materials and Methods}

\section{Animals}

Male nude mice (BALB/c-nu, 6 8 weeks) were purchased and were housed in a pathogen-free animal facility with access to water and food and allowed to eat and drink ad libitum. All of the experimental procedures were performed in accordance with protocols approved by the Institutional Animal Care and Research Advisory Committee of Tianjin Medical University.

\section{Human tissue}

Human gastric cancer tissues and paired adjacent noncancerous tissues (16 pairs, total 32 samples) were derived from patients undergoing a surgical procedure at the Tianjin Medical University Cancer Institute and Hospital (Tianjin, China). Among those patients, there were 9 men and 7 women. The average age was 64 (range from 43-79). The clinicopathological features and type of adenocarcinoma (intestinal, diffuse or mixed) of patients were listed in the Table 1. Both tumor tissues and noncancerous tissues were histologically confirmed. The pathological type of each cancer was determined to be adenocarcinoma. The study was conducted with the approval of the Ethics Committee of Tianjin Medical University Cancer Institute and Hospital and informed consent was obtained before surgery. Tissue fragments were immediately frozen in liquid nitrogen at the time of surgery and stored at $-80^{\circ} \mathrm{C}$.

\section{Cell culture}

Human gastric cell line MGC-803 and SGC-7901 were purchased from the Shanghai Institute of Cell Biology of the Chinese Academy of Sciences (Shanghai, China). MGC-803 and SGC-7901 cells were cultured in DMEM (Gibco, USA), supplemented with $10 \%$ fetal bovine serum (FBS, Gibco, USA) and $1 \%$ penicillin/ streptomycin (Solarbio, China) in a humidified incubator at $37^{\circ} \mathrm{C}$ with $5 \% \mathrm{CO}_{2}$. Cells were grown in culture dishes.

\section{RNA isolation and quantitative RT-PCR}

Total RNA was extracted from the cultured cells and tissues using TRIzol Reagent (Invitrogen, USA) following as the manufacturer's protocol. MiRNA was quantitated using Taqman microRNA probes (Applied Biosystems, Foster City, CA). RTPCR used CFX96 (Bio-Rad) as follows: $95^{\circ} \mathrm{C}$ for 30 s, 40 cycles of $95^{\circ} \mathrm{C}$ for $5 \mathrm{~s}$, and $60^{\circ} \mathrm{C}$ for $30 \mathrm{~s}$. After the reactions were completed, the cycle threshold (CT) data were determined using fixed threshold settings, and the mean CT was calculated from triplicate PCRs. A comparative CT method was used to compare each condition to the control reactions. U6 snRNA was used as an internal control of miRNAs, the HGF and VEGF mRNA levels were normalized to the corresponding housekeeping gene GAPDH. The relative amount of gene normalized to control was calculated with the equation $2^{-\triangle \mathrm{CT}}$, in which $\Delta \mathrm{CT}=$ $\mathrm{CT}_{\text {gene }}-\mathrm{CT}_{\text {control }}$. All of the reactions were performed in triplicate. Primers of HGF, VEGF and GAPDH were designed as follows:

5'-AGAAGGCTGGGGCTCATTTG-3' (GAPDH, sense);

5'-AGGGGCCATCCACAGTCTTC-3' (GAPDH, anti-sense);

5'-ACCCTGGTGTTTCACAAGCA-3' (HGF, sense);

5'-GCAAGAATTTGTGCCGGTGT-3' (HGF, antisense);

Table 1. The clinicopathological features of GC patients $(n=16)$. ECOG: The Eastern Cooperative Oncology Group; LVI: Lymphovascular invasion; PNI: Perineural invasion

\begin{tabular}{|c|c|}
\hline Characteristcs & $\begin{array}{l}\text { Number of patients (\%) } \\
n=16\end{array}$ \\
\hline Age, median(range) & $64(43-79)$ \\
\hline \multicolumn{2}{|l|}{ Sex } \\
\hline Female & $7(43.8)$ \\
\hline Male & $9(56.2)$ \\
\hline \multicolumn{2}{|l|}{ Smoking history } \\
\hline Yes & $10(62.5)$ \\
\hline No & $6(37.5)$ \\
\hline \multicolumn{2}{|l|}{ Family history } \\
\hline Yes & $4(25.0)$ \\
\hline No & $12(75.0)$ \\
\hline \multicolumn{2}{|l|}{ ECOG performance status } \\
\hline $0-1$ & $14(87.5)$ \\
\hline 2 & $2(12.5)$ \\
\hline \multicolumn{2}{|l|}{ Lauren classification } \\
\hline Intestinal & $6(37.5)$ \\
\hline Diffuse & $7(43.8)$ \\
\hline Mixed & $3(18.7)$ \\
\hline \multicolumn{2}{|l|}{ Tumor location } \\
\hline Fundus, cardia & $5(31.3)$ \\
\hline Corpus & $3(18.7)$ \\
\hline Antrum & $8(50.0)$ \\
\hline \multicolumn{2}{|l|}{ TNM classification } \\
\hline I-II & $3(18.7)$ \\
\hline III-IV & $13(81.3)$ \\
\hline \multicolumn{2}{|l|}{ LVI } \\
\hline Yes & $6(37.5)$ \\
\hline No & $10(62.5)$ \\
\hline \multicolumn{2}{|l|}{ PNI } \\
\hline Yes & $6(37.5)$ \\
\hline No & $10(62.5)$ \\
\hline \multicolumn{2}{|l|}{ Metastatic regions } \\
\hline Distant lymph node & $3(18.7)$ \\
\hline Liver & $2(12.5)$ \\
\hline Peritoneum & $1(6.25)$ \\
\hline
\end{tabular}




\section{Cellular Physiology Cell Physiol Biochem 2017;42:1670-1683 \begin{tabular}{c|c|c|} 
DOI: 10.1159/000479412 & (0 2017 The Author(s). Published by S. Karger AG, Basel \\
www.karger.com/cpb
\end{tabular}}

Si et al.: Novel Cancer Suppressive miRNAs in Gastric Carcinoma

5'-ATCTTCAAGCCATCCTGTGTGC-3' (VEGFa, sense);

5'-GCTCACCGCCTCGGCTTGT-3' (VEGFa, anti-sense).

\section{Cell transfection}

MiR-26a/b mimic and inhibitor were purchased from RiboBio with corresponding controls (RiboBio, China). MGC-803 and SGC-7901 cells were plated in six-well or other plates and transfected using lipofectamine 2000 (Invitrogen, Life Technologies) and Opti-MEM Reduced Serum Medium (Gibco, Life Technologies) 24h later according to the manufacturer's instructions. For miRNA over-expression and down-regulation, $100 \mathrm{pmol}$ miR-26a/b mimics, inhibitors and negative control were used.

The HGF overexpressing and scilencing lentivirus and the control lentivirus were bought from Santa cruz (sc-404011-LAC, sc-39554-V). For HGF overexpressing, $10^{6}$ lentivirus were added into every single well with DMEM medium, and polybrene in a MOI of 10 just as manufacturer's instructions. For HGF silencing, stable knock down of HGF was performed using short hairpin RNA (shRNA) lentiviral constructs. The lentiviral shRNA was added to cultured cells for 24 hours, and cells were selected by puromycin $(1.0 \mu \mathrm{g} /$ $\mathrm{mL}$ ) for 10-14 days. Knockdown and augment of HGF was verified by Western blot assay. Control cells were infected with non-sense control shRNA lentiviral particles (Santa Cruz Biotech).

\section{Luciferase assay}

The reporter plasmid p-MIR-HGF containing the predicted miR-26a/b targeting regions was designed by Genescript (Nanjing, China). Part of the wild type and mutated 3'-UTR of HGF was cloned immediately downstream of the firefly luciferase reporter. The $2 \mathrm{mg}$ of $\beta$-galactosidase expression vector (Ambion) was used as a transfection control. For the subsequent luciferase reporter assays, $2 \mathrm{mg}$ of firefly luciferase reporter plasmid, $2 \mathrm{mg}$ of $\beta$-galactosidase vector, and equal doses (200 pmol) of mimics, inhibitors, or scrambled negative control RNA were transfected into the prepared cells. At $24 \mathrm{~h}$ after transfection, cells were analyzed using the Dual Luciferase Assay Kit (Promega) according to the manufacturer's instructions. Each sample was prepared in triplicate and the entire experiment was repeated three times.

\section{Western blotting}

The HGF and VEGF expression was assessed by western blotting analysis, and samples were normalized to GADPH. MGC-803 and SGC-7901 cells were plated in six-well and cultured in DMEM with 10 $\%$ FBS for $24 \mathrm{~h}$. After transfection for $48 \mathrm{~h}$, about $50 \mu \mathrm{g}$ of protein was extracted from the cultured cells that were solubilized in lysis buffer. The proteins were separated by sodium dodecyl sulfate-polyacrylamide gel electrophoresis and then transferred to polyvinylidene difluoride membranes (Roche). The membranes were blocked within $2 \%$ bovine serum albumin (BSA) at room temperature for $1 \mathrm{~h}$ and incubated overnight at $4{ }^{\circ} \mathrm{C}$ with primary anti-HGF(1:500, Santa Cruz), anti-VEGF(1:200, Santa Cruz) and anti-GADPH (1:1000, Santa Cruz), respectively. The membranes subsequently washed and incubated with appropriate secondary anti-mouse/rabbit IgG (1:2000, Santa Cruz). After incubated with ECL, the protein bands were visualized.

\section{Cell proliferation assay}

The proliferative ability of MGC-803 and SGC-7901 cells after different transfection was determined by the EdU proliferation assay (RiboBio Inc.). Cells seeded in 24-well plates for $24 \mathrm{~h}$. Twenty-four hours after transfection, cells were incubated with $50 \mu \mathrm{M}$ EdU for $5 \mathrm{~h}$ and fixed within $4 \%$ paraformaldehyde for $30 \mathrm{~min}$ at room temperature(RT).Then the cells were washed in PBS twice and permeabilized using PBS containing $0.5 \%$ Triton X-100 for $10 \mathrm{~min}$. After extensive washes in PBS, the cells were incubated lucifugally in Apollo staining solution (RiboBio Inc.) for $30 \mathrm{~min}$, then repeated permeation and wash, and incubated in Hoechst 33342 (1:100; RiboBio Inc.) for another $30 \mathrm{~min}$ at RT. All of the staining was performed in triplicate.

\section{Cell migration assay}

The migration ability of MGC-803 and SGC-7901 cells after different transfection was measured by transwell assay. Twenty-four-well Boyden chambers with $8-\mu \mathrm{m}$ pore size polycarbonate membrane (Corning, NY) was used. After transfection, cells were transferred into the upper chamber of the transwell with $200 \mu \mathrm{l}$ serum-free growth medium $\left(10^{5}\right.$ cells per well). Complete medium containing $10 \%$ FBS was added to the lower chamber as a chemo-attractant. After $24 \mathrm{~h}$ of incubation at $37^{\circ} \mathrm{C}$, non-migratory cells on the upper surface of upper chamber were removed slightly by cotton swabs and cells that migrated to 


\section{Cellular Physiology Cell Physiol Biochem 2017;42:1670-1683 \\ \begin{tabular}{l|l} 
and Biochemistry Published onlIne: July 24, 2017 & $\begin{array}{l}\text { D } 2017 \text { The Author(s). Published by S. Karger AG, Basel } \\
\text { www.karger.com/cpb }\end{array}$
\end{tabular} \\ Si et al.: Novel Cancer Suppressive miRNAs in Gastric Carcinoma}

the bottom of the membrane were fixed and stained. The number of invaded cells was counted under light microscope. To minimize the bias, five randomly selected fields with $\times 200$ magnification were counted and then the average number was calculated. All experiments were performed in triplicate.

\section{Wound scratch assay}

The migration ability of MGC-803 and SGC-7901 cells was measured by wound-healing assay. Briefly, cells were seeded in six-well plates after different transfection. On the following day, when the cells were approximately $\geq 90 \%$, each well was scraped with a $20 \mu \mathrm{l}$ pipette tip to create 2 linear regions devoid of cells. Subsequently, the cells in each well were cultured with DMEM medium (Gibco, USA) containing 2\% fetal bovine serum (Gibco, USA) in a humidified incubator. We monitored wound closure at $0 \mathrm{~h}, 12 \mathrm{~h}, 18 \mathrm{~h}$, and $24 \mathrm{~h}$ after scraping. Five random non-overlapping images of each well were selected and quantitated for statistical analysis. All experiments were performed in triplicate.

\section{Immunohistochemistry assay}

Paraffin-embedded specimens of gastric cancer and the paired noncancerous tissues (16 pairs, total 32 samples) were sectioned and stained with an anti-HGF monoclonal antibody (sc-400, Santa Cruz Biotechnology) at a 1:50 dilution. The DAB system (Zhong shan jin qiao, China) was used to identify the positive staining. Five random fields were selected for each specimen.

The tumors from nude mice were fixed in 4\% paraformaldehyde, embedded in paraffin, sectioned, and then stained with DBE-conjugated anti-CD31 (Abcam). The fluorescence intensity was quantified from at least five sections.

Establishment of tumor xenograft in nude mice

MGC-803 cells treated with control lentivirus or miR-26a/b overexpressing lentivirus or HGF overexpressing lentivirus were injected subcutaneously into nude mice $\left(1 \times 10^{7}\right.$ cells for one mouse). Mice were sacrificed after 4 weeks, and the weight and diameter of tumors were recorded.

\section{The miRNA target prediction}

The miRNA target prediction and analysis were performed with the algorithms from TargetScan (http://www.targetscan.org/) PicTar (http://pictar.mdc-berlin.de/) and miRanda (http://www.microrna. $\operatorname{org} /$ ).

\section{Statistical analyses}

All statistical analyses were performed using IBM SPSS Statistics, version 22.0. All data were representative of at least three independent experiments. All the data were compared between two independent groups and analyzed by using the Student's test. Data were described with median values \pm SD. Differences were considered statistically significant for $\mathrm{P}<0.05$. In this study, “*” indicates "p $<0.05$ ", “**” indicates " $<0.01$ ", and "***" indicates "p $<0.001$ ".

\section{Results}

HGF protein levels but not mRNA levels are up-regulated in GC tissues

We first determined the expression characteristic of HGF in human GC tissues. HGF was detected using immunohistochemical (IHC) assays in 16 pairs of formalin-fixed, paraffinembedded sections of tissue specimens (total 32 samples). The results showed that HGF was primarily expressed in cancer tissues compared with corresponding noncancerous tissues and exhibited a cytoplasmic distribution (Fig. 1A). After measuring the protein levels of HGF in 16 pairs, we found that HGF protein levels were dramatically higher in GC tissues (Fig. 1B and 1C). Although HGF protein was consistently up-regulated in GC, HGF mRNA levels did not differ significantly between cancer and noncancerous tissues (Fig. 1D). This disparity between protein and mRNA strongly suggests that a post transcriptional mechanism is involved in HGF regulation. 
Fig. 1. Inverse correlation between HGF and miR-26a/b in human GC tissues. (A) Immunohistochemistry of the paraffin-embedded human gastric cancer tissues and adjacent noncancerous tissues ( $n=16$, total 32 samples). (B, C) Western blot analysis of HGF expression and quantitative analysis of HGF in GC cancer tissue and the paired adjacent noncancerous tissue $(\mathrm{n}=16)$. (D) Quantitative RT-PCR analysis of HGF mRNA levels in GC cancer tissue and the paired adjacent noncancerous tissue $(n=16)$. (E) The predicted binding sites of miR-26a/b in the mRNA of HGF. (F) Relative levels of miR-26a/b in GC serum and normal serum $(\mathrm{n}=$ 150). (G) Relative levels of miR$26 \mathrm{a} / \mathrm{b}$ in GC tissues and normal tissues $(n=16)$. NC is the paired noncancerous group of GC. ${ }^{* * *} \mathrm{p}$ $<0.001,{ }^{* *} \mathrm{p}<0.01,{ }^{*} \mathrm{p}<0.05$.

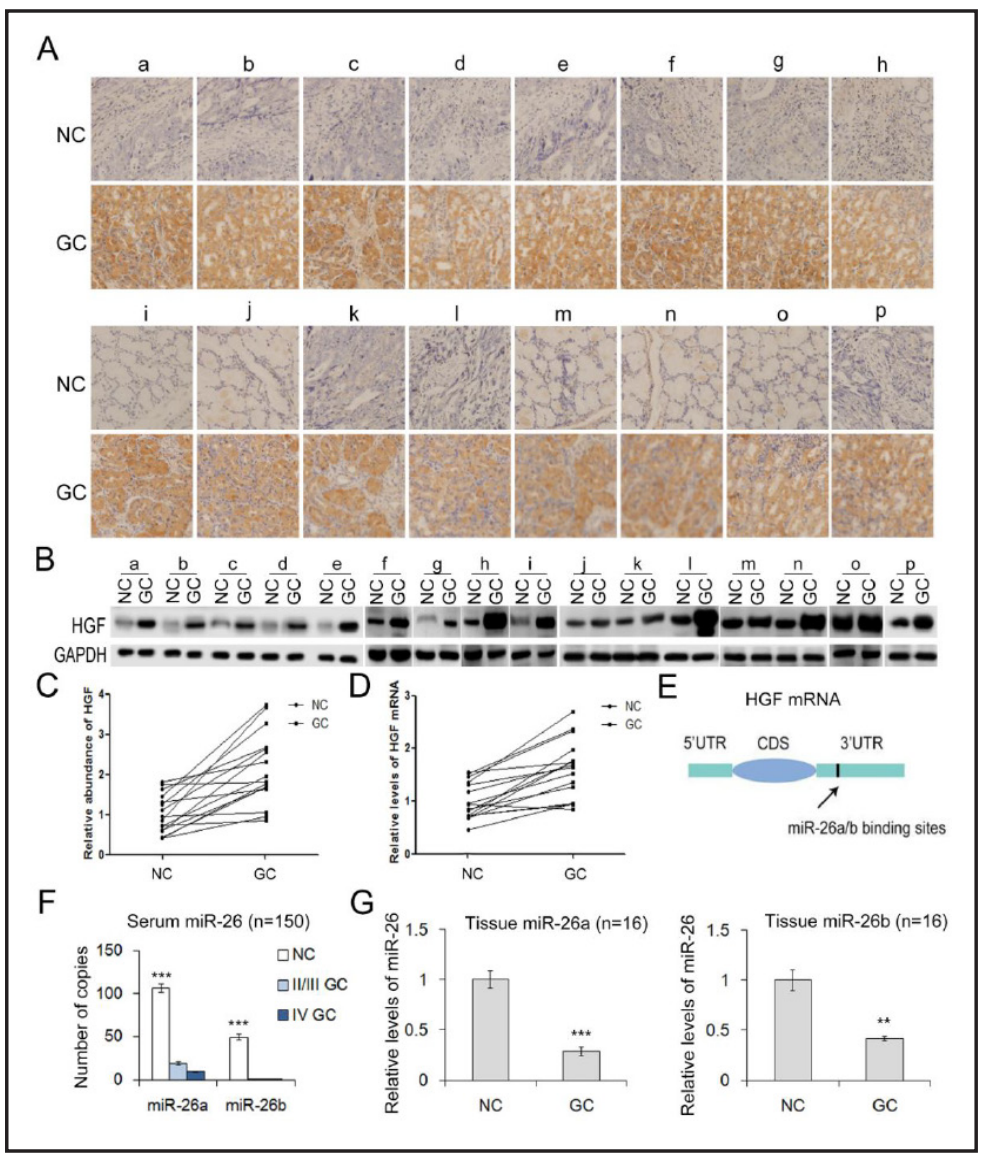

miR-26a/b are down-regulated in the serum and tissues of GC patients and act as a potential regulator of $H G F$

The miRNA-mediated specific mRNA cleavage or translational repression is one of the most important post-transcriptional regulatory mechanisms. In our study, we identified, using bioinformatics tools, that miR-26a/b directly binds to a region in the 3'UTR of HGF mRNA. The binding site in a forward position is highly conserved and is stably expressed among different species (Fig. 1E). As is shown in Fig. 2A, miR-26a/b could bind with HGF mRNA by complementary base pairing at this target region. To validate the actual relationship between miR-26a/b and HGF in GC, we explored the level of miR-26a/b in 16 pairs of GC tissues and corresponding noncancerous tissues. As a result, miR-26a/b showed an obvious decrease in tumor tissues (Fig. 1G). In addition, we also evaluated the expression level of $\mathrm{miR}-26 \mathrm{a} / \mathrm{b}$ in the serum of GC patients and found that it was similar to that in the tissues (Fig. 1F). Therefore, the levels of miR-26a/b and HGF showed an inverse correlation in GC. miR-26a/b are most likely important regulators of HGF in GC cells.

$H G F$ is validated as a direct target of miR-26a/b and correlates with the expression of VEGF

Based the prediction by the bioinformatics tools, a luciferase assay was performed to evaluate the direct interaction between miR-26a/b and HGF. The relative luciferase activity was clearly inhibited when miR-26a/b mimics were co-transfected with luciferase reporters containing the predicted binding region of the wild-type 3'UTR of HGF. However, the interaction was lost when a plasmid with a mutated sequence was used instead. Furthermore, the co-transfection of miR-26a/b inhibitors and the plasmid with the wild HGF 3'UTR resulted in a relative increase in the luciferase signal (Fig. 2B).

The same biological effect of miR-26a/b on HGF was evaluated in MGC-803 and SGC7901 cell lines. The expression of HGF was detected using Western blot. Relative levels KARGER 


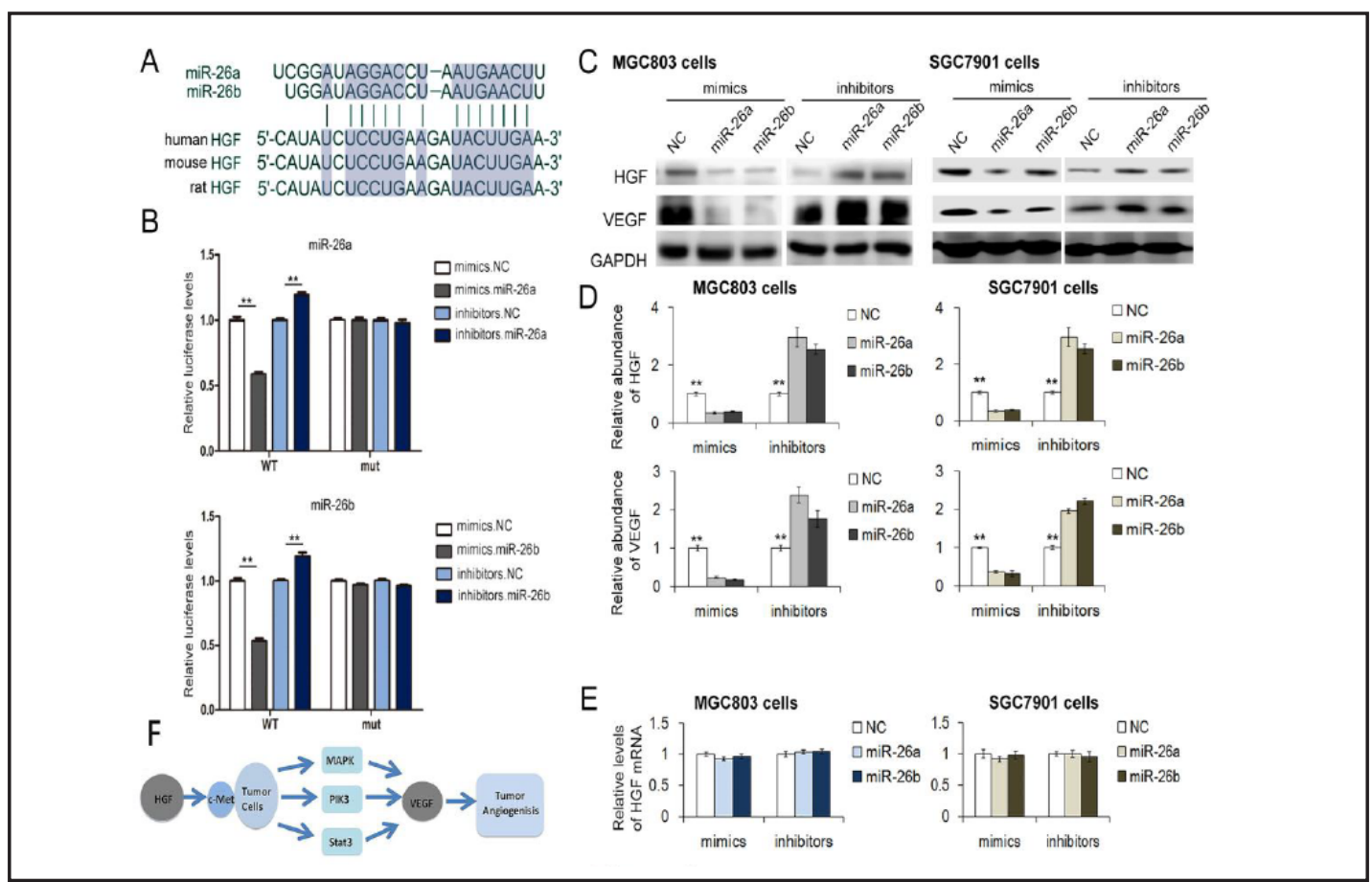

Fig. 2. HGF is a direct target of miR-26a/b and correlated related with the expression of VEGF. (A) Schematic description of the base pairing interaction between miR-26a/b and HGF mRNA. (B) Direct recognition of HGF by miR-26a/b. MGC-803 cells were co-transfected with firefly luciferase reporters containing either WT or mutant HGF 3'-UTR with miR-26a/b mimics and inhibitors. An interaction between the miR-26a/b and the target was evident. (C) The reduction of HGF expression by miR-26a/b mimics in MGC-803 cells and SGC-7901 cells, VEGF expression level decreased caused by suppression of HGF. (D) Quantitative analysis of (C). (E) Quantitative RT-PCR analysis of HGF mRNA levels in MGC-803 cells and SGC-7901 cells treated with miR-26a/b mimics and inhibitors. (F) The sketch map of HGF-VEGF pathway. HGF/c-Met signaling turns on the angiogenic switch by targeting at MAPK, PIK3 and Stat3 to up-regulate VEGF expression in the tumor cells. NC is the corresponding negative control of mimics or inhibitors. ${ }^{* *} \mathrm{p}<0.01$.

of miR-26a/b in the two cell lines were detected using qRT-PCR analysis following the transfection of mimics or inhibitors (Fig. 2C and 2D). As is shown in Fig. 2C and 2D, the overexpression of miR-26a/b by the transfection of mimics led to a clear suppression of HGF protein. Transfection of miR-26a/b inhibitors enhanced the expression of HGF in both MGC803 cells and SGC-7901 cells. Meanwhile, HGF mRNA was not changed with the transfection of mimics or inhibitors (Fig. 2E).

Previous studies have found that HGF plays a significant role in tumor angiogenesis and induces VEGF expression in tumor cells [12-15]. A sketch map about HGF and its relationship with VEGF pathway is summarized in Fig. 2F. In our study, we determined with Western blot and qRT-PCR that the expression of VEGF exhibits the same trend as HGF when miR-26a/b was overexpressed or inhibited. (Fig. 2C and 2D).

In conclusion, these results demonstrated that miR-26a/b regulated HGF by directly binding a specific region on the 3'-UTR of HGF. Meanwhile, HGF enhances the expression of VEGF as previously reported.

miR-26a/b inhibits the proliferation and migration of MGC-803 cells

We next examined the biological effects of miR-26a/b on GC cells. The effect of miR$26 \mathrm{a} / \mathrm{b}$ on the proliferation of MGC-803 cells was detected in vitro by the EdU proliferation assay (Fig. 3D and 3E). As expected, the proliferation rate in MGC-803 cells transfected with miR-26a/b mimics was significantly decreased compared with the NC mimics group. Meanwhile, the proliferation rate in MGC-803 cells transfected with miR-26a/b inhibitors 
Fig. 3. miR-26a/b regulates the proliferation and migration of MGC803 cells. (A)Validation of miR-26a/b-mediated cell migration by the scraping line method.(B) Transwell assays demonstrated that miR-26a/b suppressed the migration of MGC-803 cells. Overexpression of miR-26a/b suppressed cell migration, whereas low levels of miR-26a/b promoted MGC-803 cells migration. (C) Quantitative analysis of (B). (D) EdU assays demonstrated that miR-26a/b suppressed the proliferation of MGC803 cells. Overexpression of miR-26a/b suppressed cell proliferation, whereas low levels of miR-26a/b promoted MGC-803 cells proliferation. (E) Quantification of D. NC is the corresponding negative control of mimics or inhibitors. $* *$ $\mathrm{p}<0.01$.

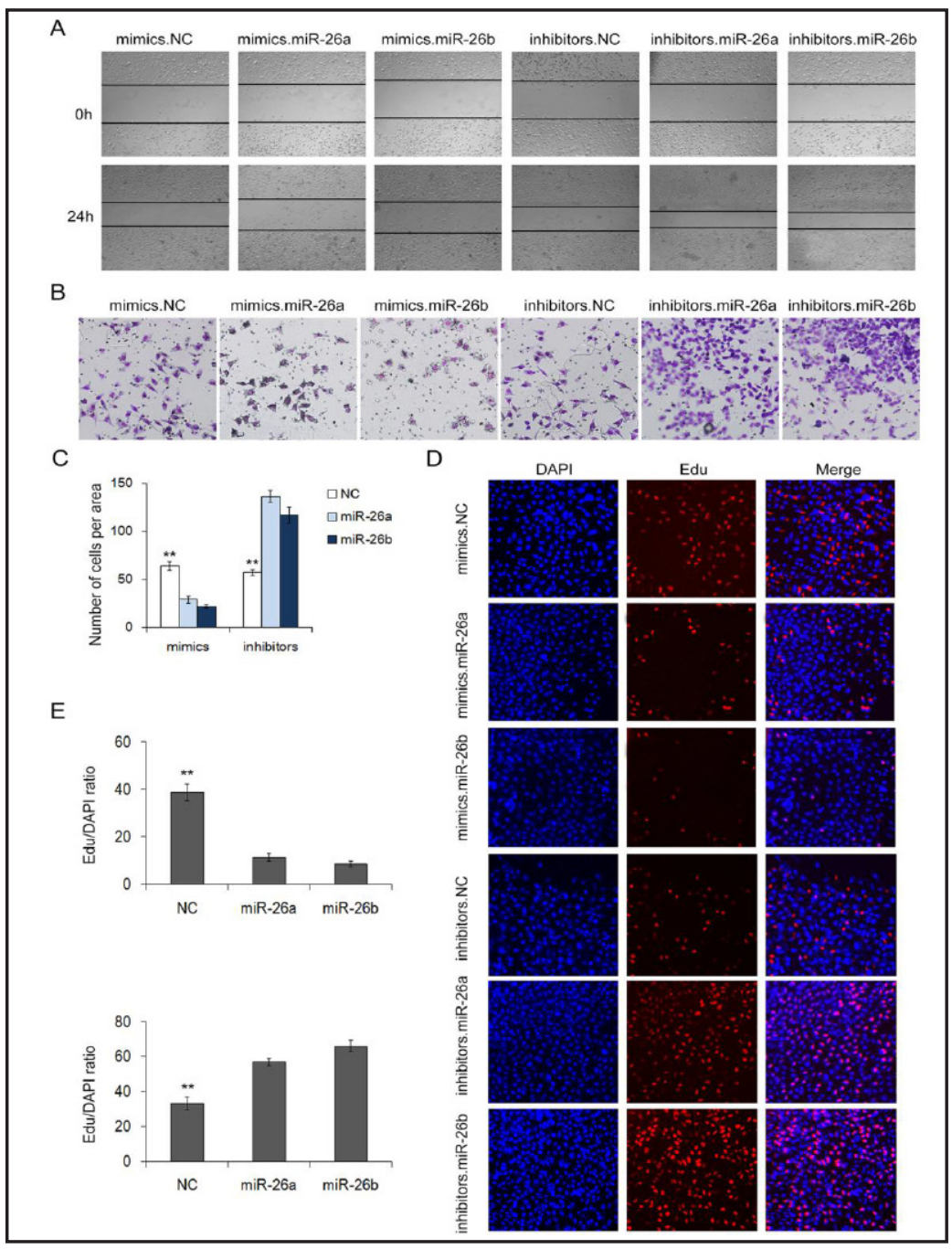

showed a sharp increase compared to the NC inhibitor group. All of these data showed that miR-26a/b could inhibit the proliferation of MGC-803 cells line.

To determine whether miR-26a/b affected cell migration, a wound healing assay (Fig. $3 \mathrm{~A}$ ) and a Transwell assay (Fig. 3B and 3C) were performed after transfection with miR$26 \mathrm{a} / \mathrm{b}$ mimics, inhibitors or miRNA NC in the MGC-803 cell line. Both the wound healing assay and the Transwell assay demonstrated that MGC-803 cells transfected with miR-26a/b mimics showed a lower migration ratio. In contrast, cell migration was strongly enhanced when cells were transfected with miR-26a/b inhibitors.

miR-26a/b inhibits the proliferation and migration of SGC-7901 cells

We examined the biological effects of miR-26a/b on the other GC cell line SGC-7901. As with the MGC-803 cells, the effect of miR-26a/b on the proliferation of SGC-7901 cells was detected in vitro by the EdU proliferation assay (Fig. 4D and 4E), and the effect on cell migration was evaluated by the wound healing assay (Fig. 4A) and the Transwell assay (Fig. 4B and 4C). The results showed that the proliferation and migration rates in SGC-7901 cells transfected with miR-26a/b mimics were significantly decreased compared with the control group. On the contrary, the proliferation and migration rates of SGC-7901 cells transfected with miR-26a/b inhibitors were obviously enhanced compared to control.

The results in Fig. 3 and Fig. 4 show that miR-26a/b are tumor suppressive miRNAs in GC lines that could suppress proliferation and migration. In other words, they play a vital role in the tumorigenesis of GC cells. 
Fig. 4. miR-26a/b regulates the proliferation and migration of SGC7901 cells. (A) Validation of miR-26a/b-mediated cell migration by the scraping line method. (B) Transwell assays demonstrated that miR$26 a / b$ suppressed the migration of SGC-7901 cells. Overexpression of miR-26a/b suppressed cell migration, while low levels of miR-26a/b promoted SGC-7901 cells migration. (C) Quantitative analysis of (B). (D) EdU assays demonstrate that miR-26a/b suppressed the proliferation of SGC-7901 cells. Overexpression of miR26a/b suppressed cell proliferation, while low levels of miR-26a/b promoted SGC-7901 cells proliferation. (E) Quantification of D. NC is the corresponding negative control of mimics or inhibitors. ${ }^{* *} \mathrm{p}<0.01$.

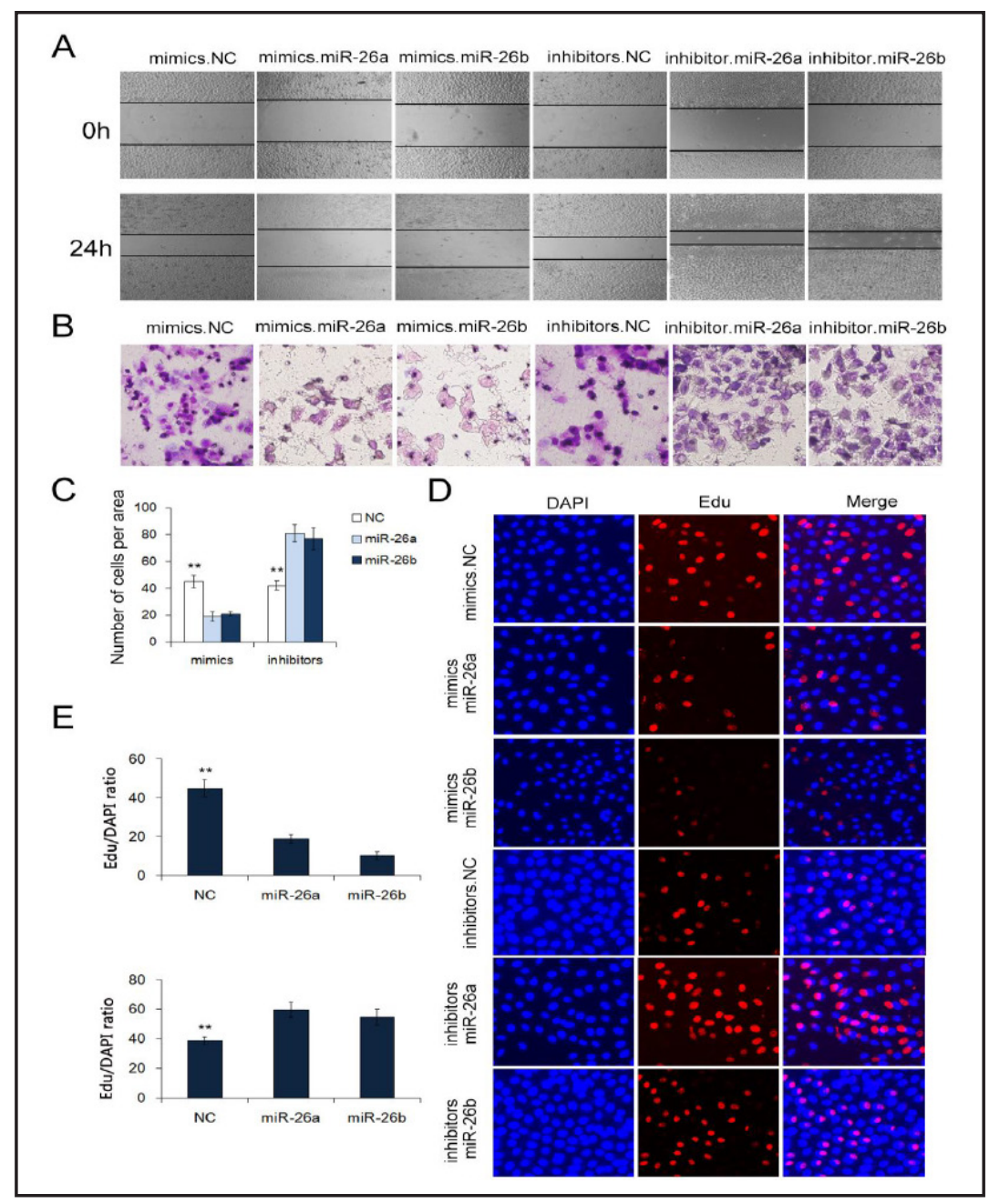

Effects of HGF overexpression and silencing on the proliferation and migration of GC cells

To further explore HGF-regulated cell proliferation and migration in GC cells, a lentiviral shRNA strategy was used to knock-down HGF in MGC-803 cells, and lentiviruses expressing the ORF of HGF were designed to overexpress HGF. The reletalive abundance of HGF was significantly reduced in MGC-803 cells transfected with shRNA and was augmented by the overexpression virus as evidenced by Western blotting and qRT-PCR (Fig. 5A and 5C). The level of HGF mRNA was consistent with the protein content (Fig. 5B). At the same time, the overexpression and silencing of the HGF caused synchronous changes in VEGF (Fig. 5D). From our experimental data, we confirmed that HGF overexpression stimulated an increase in VEGF expression.

Subsequently, the function of HGF was also measured. The EdU proliferation assay and the Transwell assay indicated that MGC-803 cells transfected with HGF shRNA had a significantly lower rate of proliferation and exhibited decreased migration compared to the control group, whereas HGF overexpression could promote proliferation (Fig. 5G and $5 \mathrm{H})$. Furthermore, the up-regulation of HGF could significantly increase cell migration significantly (Fig. 5E and 5F).

Therefore, HGF acts as a cancer promoter in GC. The dramatic up-regulation of HGF contributes to a faster rate of proliferation and a stronger migration ability in cancer cells. In addition, HGF plays an important role by cooperating with VEGF in angiogenesis. 
Fig. 5. Overexpression and silencing of HGF regulates the proliferation and migration in GC cells. (A) Silencing of HGF expression by shR$\mathrm{NA}$, overexpression of HGF using an overexpression lentivirus, MGC803 cells were transfected with HGF silencing and overexpressing lentivirus.(B,C,D) the HGF,VEGF protein expression and mRNA of HGF levels were measured. (E) Transwell assays demonstrated that overexpression of HGF strongly enhanced cell migration in MGC803 cells, whereas knockdown of HGF inhibited cell migration. (F) Quantitative analysis of (E). (G) Silencing of HGF clearly decreased the proliferation of MGC-803 cells, whereas overexpression of $\mathrm{HGF}$ promoted cell proliferation. (H) Quantitative analysis of (G). sh.NC is the negative control of shRNA of HGF. OE.NC is the negative control of HGF overexpression lentivirus. ${ }^{* *} \mathrm{p}<0.01$.
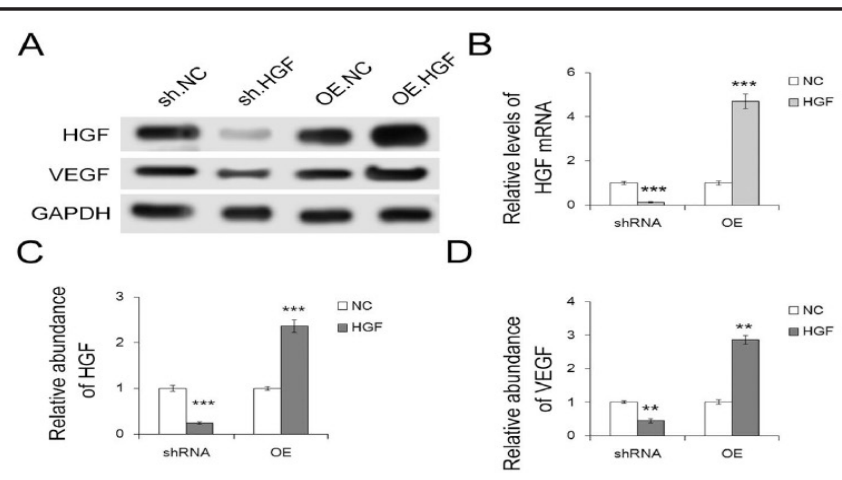

E
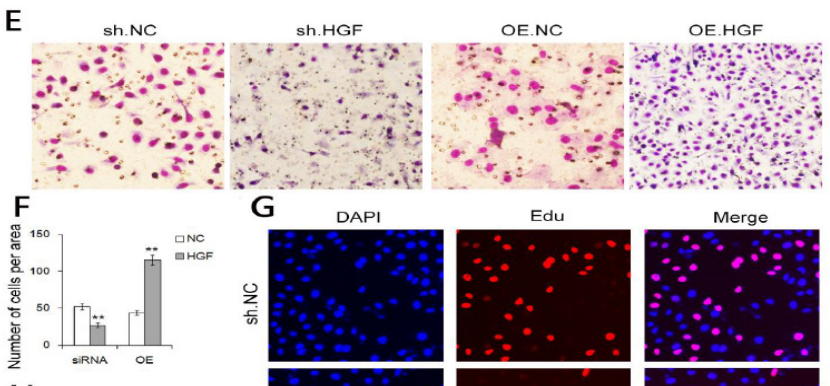

$\mathrm{H}$
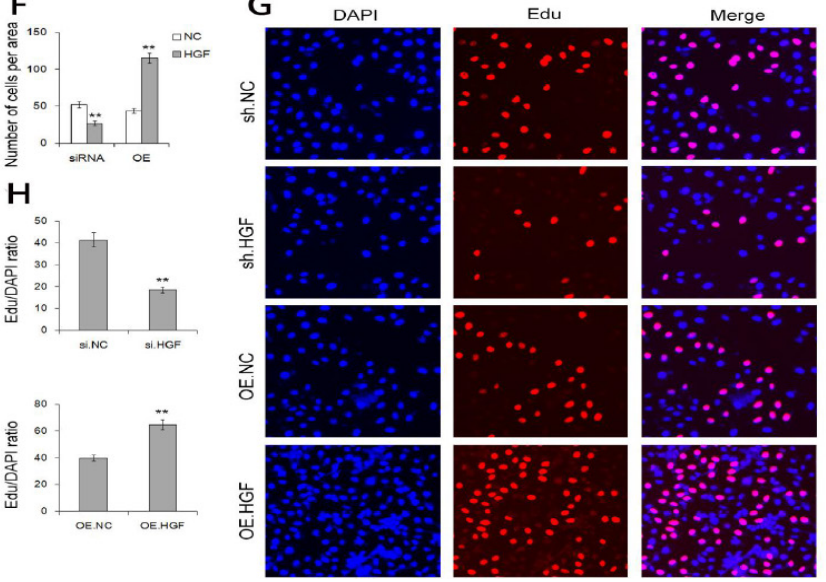

The miR-26a/b-HGF-VEGF pathway regulates tumor growth and angiogenesis in vivo

In vitro, we confirmed that the miR-26a/b-HGF-VEGF pathway at a cellular level. Then, we assessed the effects of miR-26a/b and HGF in tumor growth using a mouse implanted tumor model. MGC-803 cells were transfected with lentivirus particles to overexpress miR$26 \mathrm{a} / \mathrm{b}$ or HGF, and the cells were injected subcutaneously into the armpit of nude mice. As is displayed in Fig. 6A and 6B, overexpression of miR-26a/b obviously decreased the tumor size and weight, while the overexpression of HGF strongly boosted tumor growth. The levels of miR-26a/b and HGF were detected in the excised tumors. Our results indicated that miR$26 \mathrm{a} / \mathrm{b}$ were obviously elevated in the miR-26a/b-overexpression group, and HGF increased by nearly 4-fold in the HGF-overexpression group compared with the control group (Fig. 6C,6D and 6E). When miR-26a/b were overexpressed, HGF expression was much lower than that in the control group, which was in accordance with the results from the in vitro experiments (Fig. 6C, 6D and 6F). It is worth noting that the expression level of VEGF in the HGF-overexpression group was also distinctly raised (Fig. 6C, 6D and 6F). To further confirm that the miR-26a/b-HGF pathway up-regulates the expression of VEGF to promote angiogenesis, tumor angiogenesis was evaluated by IHC using CD31 as a vascular marker. The results showed that the relative vessel density was lower in the miR-26a/b-overexpression group than that in the control group; meanwhile, the relative vessel density in the HGFoverexpression group was much higher compared with the control group. (Fig. 6G).

These results from in vivo experiments further demonstrated that miR-26a/b act as suppression miRNAs by inhibiting HGF expression in GC. HGF promotes angiogenesis by targeting VEGF. 


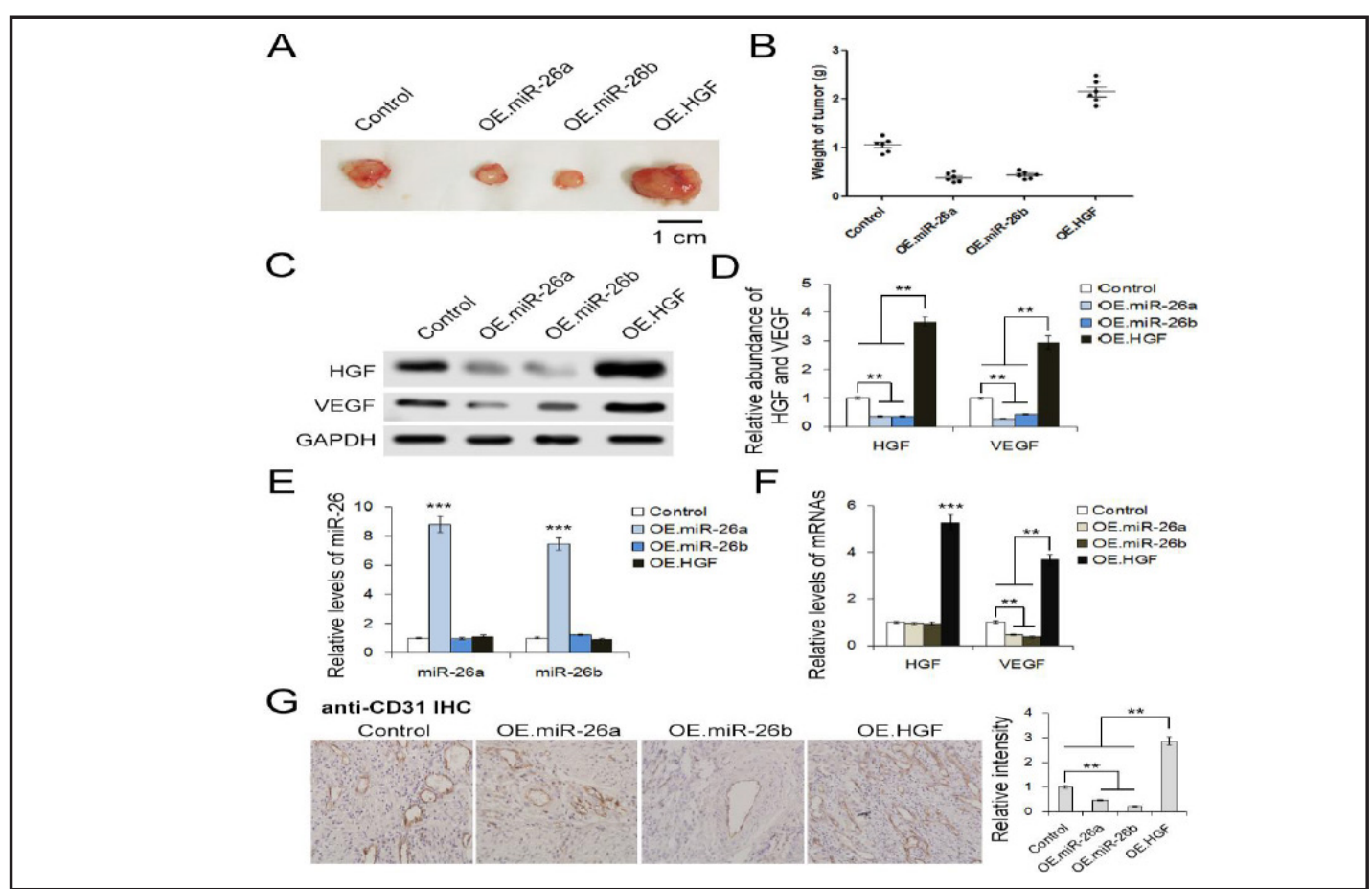

Fig. 6. The miR-26a/b-HGF-VEGF pathway regulates tumor growth and angiogenesis in vivo. (A) Representative image of tumors excised from nude mice. MGC-803 cells were treated with miR-26a/b-overexpressing lenti-virus, HGF-overexpressing lentivirus and the control lentivirus respectively, and cells were implanted in BALB/c mice. (B) Weight of tumors from mice implanted with MGC-803 cells. (C) HGF and VEGF expression in implanted tumors and western blot analysis of HGF and VEGF levels. (D) Quantitative analysis of (C). (E) Quantitative RT-PCR analysis of miR-26a/b in implanted tumors. (F) Relative levels of mRNAs of HGF and VEGF in different implanted tumors. (G) Immunohistochemical analysis of the paraffin-embedded tumor tissues from nude mice using CD31 antibody, quantitative analysis of CD31 intensity in tumor sections. ** $\mathrm{p}<0.01,{ }^{* * *} \mathrm{p}<0.001$.

\section{Discussion}

Currently, chemotherapy and radiation are the main therapeutic methods for GC, which often leads to nonspecific mass cell death and side effects [23,24]. A better comprehension of cancer, especially the molecular mechanisms that regulate tumor growth, provides novel drug targets for future clinical use. Mounting evidence has indicated that miRNAs functionally serve as oncogenes or tumor-suppressor genes and regulate multiple cellular processes that are relevant to tumorigenesis and cancer progression [25, 26]. However, there are controversies about the roles of miR-26a/b in human malignancies. Many studies have shown that miR-26a/b serve as potential tumor suppressors. For example, miR-26a was decreased in hepatocellular carcinoma and could suppress tumor angiogenesis through HGF/c-Met signaling [27]. Breast cancer also exhibited decreased expression of miR-26a, and the overexpression of this miRNA resulted in the inhibition of tumor growth and metastasis [28]. While other studies have indicated that it exhibited oncogenic properties in glioma, lung cancer, and cholangiocarcinoma $[29,30]$, the real reason for the dual effects of miR-26a/b is not yet clear, which might be in part due to organ-specific actions and the different cellular contexts of tumors. In this study, we focused on the characteristics of miR-26a/b in GC, which was based on the results of our group that previously showed that $\mathrm{miR}-26 \mathrm{a} / \mathrm{b}$ were down-regulated in the serum of GC patients [31]. In the present study, we determined that the level of miR-26a/b expression was significantly lower in GC tissue and serum than in adjacent non-carcinoma tissue and normal serum. Using in vitro and in vivo assays, we identified the tumor suppressor function of miR-26a/b in GC. By overexpressing 
and knocking down miR-26a/b, we confirmed that miR-26a/b could inhibit the proliferation and migration of MGC-803 and SGC-7901 cells in vitro as well as suppress tumor growth in vivo. Our findings suggest that miR-26a/b serve a suppressor role in GC.

Various studies have shown that PTEN, EZH2, SMAD1, CDK6, and cyclin E1 [32] are potential downstream target genes of miR-26a/b. In this study, we identified HGF as a novel and direct target of miR-26a/b. It is well known that HGF/c-Met plays an important role in most human solid tumors, and the inappropriate expression of this ligand-receptor pair is often associated with poor prognosis in $\mathrm{GC}[33,34]$. The molecular basis for the malignant potential of the HGF/c-Met signal is mostly attributed to its mitogenic and invasive properties. In our study, we first confirmed that HGF was a direct target of miR-26a/b in GC. We determined whether miR-26a/b suppresses MGC-803 and SGC-7901 cell proliferation and migration by targeting HGF, and found that the overexpression of miR-26a/b led to the suppression of HGF protein. Moreover, there was an inverse correlation between miR-26a/b expression and HGF expression in GC tissues. These results suggest that miR-26a/b inhibit GC proliferation and migration partly by targeting HGF. In addition, HGF also induces angiogenesis, which is an essential component of tumor development. VEGF is a potent agonist of angiogenesis that activates both endothelial cell proliferation and migration. The present study provides insight into the mechanisms by which HGF induces tumor angiogenesis, which not only directly induces proliferation and migration in endothelial cells but also triggers angiogenic switching in tumor cells, by activating the expression of VEGF [35]. From our results, we verified that the expression of VEGF has the same trend as HGF when overexpressing or inhibiting miR-26a/b in vitro and in vivo. In summary, miR-26a/b serve as an up-stream gene that targets the HGF-VEGF signaling pathway to promote proliferation, migration and angiogenesis in GC.

In addition, the low early diagnosis rate of GC is one of the main reasons for poor prognosis; therefore, novel biomarkers for the early detection of GC are urgently needed to reduce disease morbidity and mortality. Several studies have found that miRNAs are a promising biomarker for early diagnosis and for predicting survival in GC patients, as they are expressed in stable and robust levels in tissues and blood [36-38]. For example, Komatsu et al. [36] identified that a high miR-21 concentration in plasma was an independent prognostic factor in GC. Another study found that high expression levels of miR-20a, miR-25, miR-93, miR-130 and miR-155 in GC tissues appeared to be biomarkers for early diagnosis [38]. Substantial studies have shown that miRNAs can help diagnose early GC and have been associated with survival duration and the disease stage of GC as well as with tumor recurrence and metastasis. It is noteworthy that in our previous study, we systematically determined the expression levels of serum miRNAs in patients with GC and identified that miR-26a/b were down-regulated in GC patients [31]. Combined with the present study, we verified the lower expression levels of miR-26a/b in the serum and tissue of GC patients and further confirmed that miR-26a/b could inhibit the proliferation and migration of GC cells in vitro and in vivo. Whether miR-26a/b could also play a role as a biomarker for early diagnosis and be related with the survival of GC patients remain uncertain. A large number of patient samples in independent cohorts are required for further discussion. The potential clinical value of miR-26a/b requires intensive study to elucidate.

Overall, the current study provided new evidence that miR-26a/b are significantly down-regulated in GC and appear to function as tumor suppressors through the regulation of the HGF-VEGF signaling pathway. These results may help us understand the molecular mechanisms of GC tumorigenesis and suggest their potential as therapeutic targets of GC.

\section{Acknowledgments}

This work was supported by grants from the National Natural Science Foundation of China (No. 81372394, 81602158 and 81602156) and Tianjin Health and Family Planning Commission Foundation of Science and Technology (15KG142). This work was also supported by CSCO-Merck Serono Oncology Research Fund (Y-MX2015-092) and Doctoral 


\section{Cellular Physiology Cell Physiol Biochem 2017;42:1670-1683

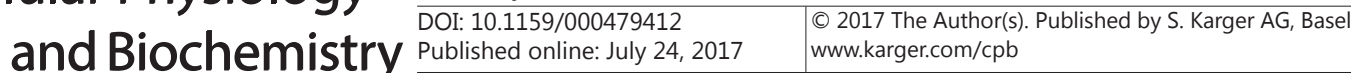

Si et al.: Novel Cancer Suppressive miRNAs in Gastric Carcinoma

foundation of Tianjin Medical University Cancer Institute and Hospital (B1502). The funders had no role in study design; in collection, analysis, and interpretation of data; in the writing of the report; and in the decision to submit this article for publication.

\section{Disclosure Statement}

The authors declare no conflict of interest.

\section{References}

1 Fitzmaurice C, Dicker D, Pain A: The Global Burden of Cancer 2013. JAMA Oncol 2015;1:505-527

2 Zhang XY, Zhang PY: Gastric cancer: somatic genetics as a guide to therapy. J Med Genet 2016;4:305-312.

-3 Toiyama Y, Tanaka K, Kitajima T, Shimura T, Imaoka H, Mori K: Serum angiopoietin-like protein 2 as a potential biomarker for diagnosis, early recurrence and prognosis in gastric cancer patients. Carcinogenesis 2015;36:1474-1483.

-4 Wang QX, Zhu YQ Zhang H, Xiao J: Altered MiRNA expression in gastric cancer: a systematic review and meta-analysis. Cell Physiol Biochem 2015;35:933-944.

-5 Noguchi E, Saito N, Kobayashi M, Kameoka S: Clinical significance of hepatocyte growth factor/c-Met expression in the assessment of gastric cancer progression. Mol Med Rep 2015;11:3423-3431.

-6 Riquelme I, Saavedra K, Espinoza JA, Weber H, García P, Nervi B, Garrido M, Corvalán AH, Roa JC, Bizama C: Molecular classification of gastric cancer: Towards a pathway-driven targeted therapy. Oncotarget 2015;6:24750-24779.

7 Joo MK, Park JJ, Chun HJ: Recent updates of precision therapy for gastric cancer: Towards optimal tailored management. World J Gastroenterol 2016;22:4638-4650.

8 Gherardi E, Birchmeier W, Birchmeier C, Vande Woude G: Targeting MET in cancer: rationale and progress. Nat. Rev. Cancer 2012;12:89-103.

-9 Wu CW, Li AF, Chi CW, Chung WW, Liu TY, Lui WY, P'eng FK: Hepatocyte growth factor and Met/HGF receptors in patients with gastric adenocarcinoma. Oncol Rep 1998;5:817-822.

10 Kang YK, Muro K, Ryu MH, Yasui H, Nishina T, Ryoo BY, Kamiya Y, Akinaga S, Boku N: A phase II trial of a selective c-Met inhibitor tivantinib (ARQ 197) monotherapy as a second- or third-line therapy in the patients with metastatic gastric cancer. Invest New Drugs 2014;32:355-361.

11 Li K, Li J: Current Molecular Targeted Therapy in Advanced Gastric Cancer: A Comprehensive Review of Therapeutic Mechanism, Clinical Trials, and Practical Application. Gastroenterol Res Pract 2016;2016:4105615.

12 Li L, Jiang X, Zhang Q Dong X, Gao Y, He Y, Qiao H, Xie F, Xie X, Sun X: Neuropilin-1 is associated with clinicopathology of gastric cancer and contributes to cell proliferation and migration as multifunctional co-receptors. J Exp Clin Cancer Res 2016;35:16.

13 Koh SA, Kim MK, Lee KH, Kim SW, Kim JR: RhoGDI2 is associated with HGF-mediated tumor invasion through VEGF in stomach cancer. Clin Exp Metastasis 2014;31:805-815.

14 Villaume K, Blanc M, Gouysse G, Walter T, Couderc C, Nejjari M, Vercherat C, Cordier-Bussat M, Roche C, Scoazec JY: VEGF secretion by neuroendocrine tumor cells is inhibited by octreotide and by inhibitors of the PI3K/AKT/mTOR pathway. Neuro-endocrinology 2010;91:268-278.

-15 Fujita H, Miyadera K, Kato M, Fujioka Y, Ochiiwa H, Huang J, Ito K, Aoyagi Y, Takenaka T, Suzuki T, Ito S, Hashimoto A, Suefuji T, Egami K, Kazuno H, Suda Y, Nishio K, Yonekura K: The Novel VEGF Receptor/METTargeted Kinase Inhibitor TAS-115 Has Marked In Vivo Antitumor Properties and a Favorable Tolerability Profile. Mol Cancer Ther 2013;12:2685-2696.

16 Shah MY, Ferrajoli A, Sood AK, Lopez-Berestein G, Calin GA: microRNA Therapeutics in Cancer - An Emerging Concept. E Bio Medicine 2016;12:34-42.

17 Zhou Q, Zheng X, Chen L, Xu B, Yang X, Jiang J, Wu C: Smad2/3/4 Pathway Contributes to TGF- $\beta$-Induced MiRNA-181b Expression to Promote Gastric Cancer Metastasis by Targeting Timp3. Cell Physiol Biochem 2016;39:453-466.

18 Xiang XJ, Deng J, Liu YW, Wan LY, Feng M, Chen J, Xiong JP: MiR-1271 Inhibits Cell Proliferation, Invasion and EMT in Gastric Cancer by Targeting FOXQ1. Cell Physiol Biochem 2015;36:1382-1394. 


\section{Cellular Physiology Cell Physiol Biochem 2017;42:1670-1683

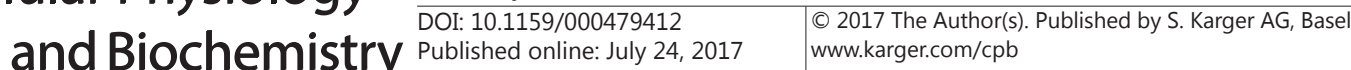

19 Li X, Li H, Zhang R, Liu J, Liu J: MicroRNA-449a inhibits proliferation and induces apoptosis by directly repressing E2F3 in gastric cancer. Cell Physiol Biochem 2015;35:2033-2042.

20 Wen L, Cheng F, Zhou Y, Yin C: MiR-26a Enhances the Sensitivity of Gastric Cancer Cells to Cisplatin by Targeting NRAS and E2F2. Saudi J Gastroenterol 2015;21:313-319.

-21 Qiu X, Zhang J, Shi W, Liu S, Kang M, Chu H, Wu D, Tong N, Gong W, Tao G, Zhao Q Qiang F, Zhu H, Wu Q, Wang M, Zhang Z: Circulating MicroRNA-26a in Plasma and Its Potential Diagnostic Value in Gastric Cancer. PLoS One DOI:e0151345.

-22 Tsai MM, Huang HW, Wang CS, Lee KF, Tsai CY, Lu PH, Chi HC, Lin YH, Kuo LM, Lin KH: MicroRNA-26b inhibits tumor metastasis by targeting the KPNA2/c-jun pathway in human gastric cancer. Oncotarget 2016;7:39511-39526.

23 Manyam BV, Mallick IH, Abdel-Wahab MM, Reddy CA, Remzi FH, Kalady MF, Lavery I, Koyfman SA: The impact of preoperative radiation therapy on locoregional recurrence in patients with stage IV rectal cancer treated with definitive surgical resection and contemporary chemotherapy. J Gastrointest Surg 2015;19:1676-1683.

24 Li S, Zhang H, Ning T, Wang X, Liu R, Yang H, Han Y, Deng T, Zhou L, Zhang L, Bai M, Wang X, Ge S, Ying G, Ba Y: MiR-520b/e Regulates Proliferation and Migration by Simultaneously Targeting EGFR in Gastric Cancer. Cell Physiol Biochem 2016;40:1303-1315.

25 Wang B, Yang H, Shen L, Wang J, Pu W, Chen Z, Shen X, Fu J, Zhuang Z: Rs56288038 (C/G) in 3'UTR of IRF-1 Regulated by MiR-502-5p Promotes Gastric Cancer Development. Cell Physiol Biochem 2016;40:391-399.

26 Zhu P, Zhang J, Zhu J, Shi J, Zhu Q, Gao Y: MiR-429 Induces Gastric Carcinoma Cell Apoptosis Through Bcl-2. Cell Physiol Biochem 2015;37:1572-1580.

27 Yang X, Zhang XF, Lu X, Jia HL, Liang L, Dong QZ, Ye QH, Qin LX: MicroRNA-26a suppresses angiogenesis in human hepatocellular carcinoma by targeting hepatocyte growth factorcMet pathway. Hepatology 2014;59:1874-1885.

28 Lu J, He ML, Wang L, Chen Y, Liu X, Dong Q Chen YC, Peng Y, Yao KT, Kung HF, Li XP: MiR-26a inhibits cell growth and tumorigenesis of nasopharyngeal carcinoma through repression of EZH2. Cancer Res 2011;71:225-233.

29 Zhang J, Han C, Wu T: MicroRNA-26a promotes cholangiocarcinoma growth by activating beta-catenin. Gastroenterology 2012;143:246-256.

-30 Liu B, Wu X, Liu B, Wang C, Liu Y, Zhou Q, Xu K: MiR-26a enhances metastasis potential of lung cancer cells via AKT pathway by targeting PTEN. Biochim Biophys Acta 2012;1822:1692-1704.

- 31 Liu R, Zhang C, Hu Z, Li G, Wang C, Yang C, Huang D, Chen X, Zhang H, Zhuang R, Deng T, Liu H, Yin J, Wang S, Zen K, Ba Y, Zhang CYZ: A five-microRNA signature identified from genome wide serum microRNA expression profiling serves as a fingerprint for gastric cancer diagnosis. Eur J Cancer 2011;47:784-791.

-32 Zhu Y, Lu Y, Zhang Q, Liu JJ, Li TJ, Yang JR, Zeng C, Zhuang SM: MicroRNA-26a/b and their host genes cooperate to inhibit the G1/S transition by activating the pRb protein. Nucleic Acids Res 2012;40:46154625 .

33 Sotoudeh K, Hashemi F, Madjd Z, Sadeghipour A, Molanaei S, Kalantary E: The clinicopathologic association of c-Met overexpression in Iranian gastric carcinomas: an immunohistoehemical study of tissue microarrays. Diagn Pathol 2012;7:57.

-34 Ha SY, Lee J, Kang SY, Do IG, Ahn S, Park JO, Kang WK, Choi MG, Sohn TS, Bae JM, Kim S, Kim M, Kim S, Park $\mathrm{CK}$, Ignatius Ou SH, Kim KM: Met overexpression assessed by new interpretation method predicts gene amplification and poor survival in advanced gastric carcinomas. Mod Pathol 2013;26:1632 -1641.

-35 Zhang YW, Su Y, Volpert OV, Vande Woude GF: Hepatocyte growth factor/scatter factor mediates angiogenesis through positive VEGF and negative thrombospondin 1 regulation. Proc Natl Acad Sci USA 2003;100:12718-12723.

-36 Komatsu S, Ichikawa D, Tsujiura M, Konishi H, Takeshita H, Nagata H, Kawaguchi T, Hirajima S, Arita T, Shiozaki A, Kubota T, Fujiwara H, Okamoto K,Otsuji E: Prognostic impact of circulating miR-21 in the plasma of patients with gastric carcinoma. Anticancer Res 2013;75:271-276.

37 Hou CG, Luo XY, Li G: Diagnostic and Prognostic Value of Serum MicroRNA-206 in Patients with Gastric Cancer. Cell Physiol Biochem 2016;39:1512-1520.

- 38 Kim BH, Hong SW, Kim A, Choi SH, Yoon SO: Prognostic and diagnosis implications for high expression of oncogenic microRNAs in advanced gastric carcinoma. J Surg Oncol 2013;107:505-510. 